Revue d'histoire de l'Amérique française

DEVVUE D.HISTOIRE DE L'AMÉRIQUE FRANÇAISE

\title{
Correspondance autour de la question scolaire du Nouveau-Brunswick 1873 (suite et fin)
}

\section{Lionel Groulx}

Volume 4, numéro 4, mars 1951

URI : https://id.erudit.org/iderudit/801672ar

DOI : https://doi.org/10.7202/801672ar

Aller au sommaire du numéro

Éditeur(s)

Institut d'histoire de l'Amérique française

ISSN

0035-2357 (imprimé)

1492-1383 (numérique)

Découvrir la revue

Citer ce document

Groulx, L. (1951). Correspondance autour de la question scolaire du

Nouveau-Brunswick 1873 (suite et fin). Revue d'histoire de l'Amérique française,

4(4), 568-575. https://doi.org/10.7202/801672ar d'utilisation que vous pouvez consulter en ligne.

https://apropos.erudit.org/fr/usagers/politique-dutilisation/ 


\title{
DOCUMENTS INÉDITS
}

\section{CORRESPONDANCE AUTOUR DE LA QUESTION SCOLAIRE DU NOUVEAU-BRUNSWICK 1873}

\author{
(suite et fin)*
}

Cher Monsieur,

Ottawa, 8 avril 1873

Nous suivons avec beaucoup d'intérêt la nouvelle lutte qui vient de commencer entre le Nouveau Monde et la Minerve. Le numéro d'hier soir a été lu avec avidité et tout le monde a reconnu que c'étaient deux maîtresarticles que ceux qu'il contenait. Pour ce qui est du Séminaire de Ste-Thérèse, Archambault dit qu'il avait été décidé avant la retraite de Chauveau qu'il y aurait une augmentation d'octroi; mais que l'Ordre en Conseil fût passé par le nouveau ministère. Il dit de plus que le Séminaire ne l'a appris que de la bouche de Ouimet à son retour de St-Jérôme. Il me semble que cela ne veut pas dire que Chapleau ne le leur avait pas dit auparavant, qu'il ne s'en était pas attribué tout le mérite ou que l'on y a pas trouvé une raison suffisante de soutenir le ministère dans la personne de son solliciteur-général.

Quant aux tours de la Minerve, ils sont bien usés et tous admettent que cela est méprisable, misérable et ne mérite que le dédain. Votre réponse a tourné tous les rieurs de votre côté.

Si vous voulez mettre Chapleau mal à l'aise, parlez de vos collègues et de la responsabilité du gouvernement local pour ses sottises, puisque le premier ministre y assistait et sanctionnait tout par sa présence. C'est le moyen de mettre la guerre dans le camp.

Nous saurons probablement jeudi si l'amnistie sera accordée à Riel et aux autres et si Manitoba recevra des better terms.

Vous pouvez annoncer que la Gazette officielle de samedi contiendra une proclamation constituant le pénitencier pour la province de Québec.

Je vous transmets comme pièces justificatives deux reçus pour un montant de $\$ 27.00$ que j'ai payées pour un mois de pension (repas seulement) et le loyer d'une chambre. C'est le montant que je vous demandais pour le 5 et que j'ai pris sur les $\$ 30.00$ reçues la semaine dernière. Arrangez cela comme il vous plaira dans vos livres.

* Voir: Reoue d'Histoire de l'Amerique française, IV, no 2 (septembre, 1950) : 268-275; IV, no 3 (décembre, 1950): 427-431. 
Ne comptez en aucune manière sur Lanthier. C'est une des âmes damnées de la Minerve et qui vendrait son âme pour son canal de la rive nord qui est une absurdité et une folie destinée à enrichir surtout Lanthier, Baillargé, Etc. qui veulent faire un job sur la rive nord comme ils accusent les seigneurs de Beauharnois d'en avoir fait un sur la rive sud.

MM. Masson et Bellerose vous présentent leurs amitiés.

Veuillez faire mes compliments à MM Lamarche et Laferrière, Trudel et Taillon, Etc.

Tout à vous.

C. Beausoleil

Cher monsieur,

Ottawa, 8 mai 1873.

Je m'attendais toujours à recevoir des nouvelles de la réunion des évêques et de leur décision à l'égard de la question des Écoles. Mercier m'a dit qu'ils avaient résolu d'ajourner toute action jusqu'au concile qui doit se réunir le 18 à Québec, tandis que M. l'abbé Michaud m'a affirmé que la dernière opinion des officiers en loi avait changé les dispositions de Mgr Sweeney et qu'il allait revenir à Ottawa et presser le Parlement de venir au secours de ses ouailles. Pourriez-vous me dire ce qu'il y a de vrai dans ces diverses assertions? J'ai revu Royal ce matin. Il m'a parlé de conversations qu'il a eues avec Rivard, M. Cherrier et autres sur l'avenir du NouveauMonde, la nécessité d'en faire un journal laique et plus exclusivement politique et de certaines rumeurs qui avaient cours sur son compte et sur ses projets. Je n'ai pas pu saisir parfaitement sa pensée ni les raisons qui l'ont empêché d'assister à la réunion des actionnaires qui a lieu aujourd'hui. J'ai bien hâte aussi de savoir ce qui est sorti de la présente réunion, des résolutions qui ont pu être adoptées, et des propositions soumises. J'espère que vous avez réussi à créer la combinaison sur laquelle vous comptiez depuis quelques mois.

Il y a plus de quinze jours que je n'ai pas reçu le Nouveau-Monde, ce qui est très incommode. Veuillez présenter mes amitiés à $M$. le chanoine Lamarche et à $M$. Laferrière et me croire

$$
\begin{aligned}
& \text { Votre tout dévoué } \\
& \text { C. BeausolenL }
\end{aligned}
$$

Mon cher Monsieur,

Ottawa, 12 mai 1873.

Je vous avouerai que je suis profondément indigné et découragé à la vue de la conduite de nos députés catholiques conservateurs du Bas-Canada. Je vous dis dans ma correspondance qu'il y a eu samedi un caucus de ces députés. Il était convoqué par Mgr Sweeney qui était venu leur demander de signer un mémoire au gouvernement pour lui demander le désaveu des dernières lois rétroactives et aggravantes pour ses ouailles, avec menace de voter aussi en faveur de cette mesure si elle était amenée par l'Opposition. 
Dès que cette pièce eût été lue, il s'éleva une tempéte. M. Baby se leva le premier pour repousser la proposition et dire qu'avant tout il ne fallait point embarrasser le gouvernement dans les circonstances actuelles et qu'il fallait plus attendre de la douceur, de la patience et des concessions que de toute mesure de violence comme celle-ci. Ross, appuya ce raisonnement, qui fut combattu, mais faiblement par M. Masson, qui déclara lui aussi qu'il n'était pas pret à signer. O'Reilly, Ryan et Fortin s'opposèrent aussi à toute initiative qui pourrait mettre en danger le ministère et le débat s'échauffant, Mgr Sweeney quitta la ville disant qu'il était venu chercher du secours et non point semer la division parmi les catholiques; que la province de Québec était le seul secours humain sur lequel il avait cru pouvoir compter et que si elle l'abandonnait il se confierait à lui seul.

Bellerose n'était pas présent, mais il dit ensuite à l'évêque qu'il marcherait en avant si la question était amenée sur le tapis. Il croit néanmoins qu'un mot des évêques aurait tout décidé, puisque Langevin se voyant appuyé et poussé au pied du mur aurait comme Tilley offert sa résignation et l'aurait emporté indubitablement puisqu'il se trouve à la tête de la seconde province de la Puissance.

Voilà done les hommes à qui le clergé a eu confiance, qui ont vécu de la religion, qui ont exploité l'influence ecclésiastique et qui on retour de vingt ans de services et de pouvoir ne savent que reculer et trahir les intérêts dont ils se disaient les conservateurs; voilà les hommes que le clergé a élus et qu'il élira encore sans doute dans cinq ans en récompense de leur zèle et de leur dévouement religieux.

Dans l'avant-midi il y avait eu un caucus de toute l'Opposition. M. McKenzie reconnut l'injustice de la loi du N.B. et déclara qu'il fallait chercher et trouver les moyens de la réparer, mais sans trop nuire au parti réformiste d'Ontario. Holton reprit qu'en effet il ne fallait pas mettre les amis d'Ontario dans une fausse position.

Dorion dit que la question était pressante et qu'il fallait une solution, tandis que Jetté appuyé de Mercier, déclara que les catholiques de Québec n'entendront point voir leurs droits de catholiques méconnus dans les autres provinces. Les catholiques sont solidaires dans toute la Puissance et qui attaque les droits d'un seul d'entre eux les attaque tous. Que feraient les protestants si leurs corélyonnaires étaient ainsi maltraités dans la province de Québec. Auraient-ils beaucoup de scrupules à intervenir; assurément non. Eh! bien nous ne ferons pas moins que les protestans et nous ne voterons pas attachés à un parti qui craindra de se compromettre pour.la justice. finale.

Il doit y avoir une nouvelle réunion aujourd'hui pour une décision

Voilà exactement ce qui s'est passé samedi et ce que l'on avait voulu tenir secret. Mais vous savez que les secrets de coulisse sont faits pour être divulgués. Ainsi les renseignements qui précèdent me viennent-ils de différentes sources également sûres et authentiques.

Vous avez dans ce qui précède une juste idée de la situation. Mgr 
Sweeney est découragé. Il voulait partir ce soir. Je ne sais si l'Opposition aura réussi à le retenir.

Je vous remercie beaucoup des renseignements que vous m'avez donnés sur la dernière assemblée des actionnaires. Je suppose qu'il n'y a pas eu beaucoup de discussions, s'il y avait si peu d'actionnaires présents. Je serai pret à partir samedi, si vous le jugez bon et si l'amnistie ou la question des Écoles ne doivent pas arriver après cette da te, comme il y a tout lieu de le croire. Je serai donc au bureau lundi matin. Veuillez done m'envoyer ce qu'il me faut pour payer un mois de pension et de chambre finissant le 5 courant $=\$ 27.00$ et 12 jours de l'un et de l'autre $\$ 10.80$ afin que je puisse régler avant de partir.

Si vous pouviez m'obtenir un billet de passage soit de la ligne de steamers de Toronto soit de celle d'Outaouais, cela épargnerait le prix du voyage entre Ottawa et Montréal qu'il faut payer à bord des chars et cela en vaudrait la peine, même s'il fallait retarder d'une journée.

Veuillez faire mes amitiés à M. le chanoine Lamarche, à M. Laferrière et me croire

Votre ami dévoué

C. Beausoleil

Privée

Ottawa, 11 novembre 1873.

Mon cher Mr. Desjardins,

Je m'attendais de partir ce soir pour Montréal, mais pour attendre le vote et surveiller les événements, je dois rester ici quelques jours encore. La chute du ministère est indubitable, et il importe que dans la formation du nouveau gouvernement il y ait place pour nos hommes et pour nos principes. L'opposition est toujours disposée à laisser un portefeuille à notre disposition et je crois qu'il serait temps de voir à jeter les yeux sur quelqu'un. Je suppose bien qu'il ne peut plus être question de Masson après la lâcheté qu'il vient de commettre et le coup de pied d'ane qu'il nous a donné quand nous étions terrassé. C'est d'ailleurs un homme qui n'a aucune fermeté et qui a retiré la parole qu'il avait donnée à Mgr Taché de voter contre le gouvernement si l'amnistie n'était pas accordée. Il m'avait déclaré que c'était là le prix de son appui, et encore une fois, il a quitté sa position. C'est bien dommage qu'il se conduise d'une façon aussi bizarre. J'avais en lui une confiance illimitée qui a complètement disparu. De Bellerose, le moins il sera dit le mieux (sic) et ce n'est pas d'un homme qui se parjure publiquement pour nous faire tort que nous avons besoin dans le gouvernement. Il est parfaitement décidé que M. Dorion n'entrera pas dans le Cabinet. M. Jetté déclare pour sa part, et tous ses amis sont d'accord sur ce point, qu'il ne formera point partie d'un gouvernement qui n'accordera pas l'amnistie et qui ne règlera point la question des Écoles. C'est lui qui est porté en avant par la députation et il désire ardemment faire une alliance avec le Nouveau Monde qui aurait pour base le règlement des questions ci-dessus et de toutes les difficultés dont Mgr de Montréal a tant souffert depuis dix ans. 
Enfin notre ami Piché serait accepté par plusieurs députés conservateurs qui ne demanderaient pas mieux que d'avoir un prétexte pour rester ministériels. Voyez donc ce qu'il y aurait à faire sous ces circonstances. C'est une occasion que nous ne devons pas perdre puisqu'elle s'offre, d'elle-même, tout naturellement, et je dirais providentiellement. J'ai averti M. Morin que je ne pouvais lui donner une réponse définitive avant le premier vote. Si les choses ne vont pas aussi bien que je l'espère et que MM. les Directeurs ne jugent pas à propos d'améliorer suffisamment mes fonctions, je me verrai à regret obligé de me séparer de vous mais non de la cause que nous avons défendue ensemble.

Bravo! Vous avez éreinté ce pauvre Victor Hudon. L'exécution est parfaite et le succès est complet. L'article sur les vrais conservateurs est aussi excellent et produit un bon effet. Soyez assuré que je n'ai fait qu'insinuer la vérité sur le comté de Laval et que c'est une élection positivement hideuse. Vous devez en avoir appris des nouvelles particulières beaucoup plus colorées que celles que je vous ai données.

Mes amitiés à M. Lamarche, à M. Hudon et aux amis.

Tout à vous,

C. BeAusoleil

\section{APPENDICE}

Le Père Paul Desjardins, s.j., qui nous a fourni l'une des lettres de Cléophas Beausoleil, celle du 8 mai 187s, nous envoie, en même temps, une lettre de Messire Godefroy Lamarche, un extrait de lettre de M. l'abbé Joseph-Uldéric Leclerc, curé de Saint-Vincent-de-Paul à $M$. Alphonse Desjardins et aussi deux extraits de La Lanterne, d'Arthur Buies, qui ont également trait au róle du Chanoine Lamarche au Nouveau-Monde. Ces extraits de Buies sont empruntés à la réédition partielle de La Lanterne, publiée en 1884. La date exacte des citations manque, nous avertit le Père Desjardins, mais il suffit de rappeler que ce journal eut vingt-sept numéros, fin-septembre 1868 à mars 1869, et que le Nouveau-Monde lui servit souvent de cible.

Nous publions la lettre et les extraits en appendice à cette correspondance. Et nous en remercions le Père Desjardins. Ces pièces jettent un peu de lumière sur des controverses qui, jadis, ont fait couler beaucoup d'encre dans la province de Québec. Documentation qui pourra servir à l'histoire des idées.

La Direction 
(M. Le chanoine G. Lamarche à Alphonse Desjardins)

Rome, Piazza del Monte di Pieta No 30 le 14 février 1877

Cher ami,

Permettez-moi de vous remercier pour l'envoi du Nouveau-Monde et de la Revue (Canadienne?), que je reçois toujours avec une régularité parfaite. Vous m'obligez d'autant plus par là, que les questions traitees par le $N$. Monde sont ici plus bralantes. Vous ne sauriez croire tout ce que j'y ai trouvé pour soutenir la bonne cause auprès des Cardinaux. Le Cardinal Oreglia surtout connaît le Nouveau-Monde comme nous et sait par cœur toute son histoire. C'est, je pense, un grand bonheur; car, la Congrégation n'a pas de membre plus actif que cet homme distingué.

Je n'ai pas besoin de vous dire ce que je fais ici, puisque j'ai entrepris d'y faire l'histoire complète de nos luttes contre les libéraux et les gallicans de toutes nuances. Tandis que j'y suis, je parle librement et, grâce à Dieu, on m'écoute. On a beau se faire donner la purgation canonique des titres, pour se ramasser un peu, je continue à bûcher comme un sourd et à demander justice pour nos principes outragés. Ma maxime à l'égard de nos adversaires, grands et petits, est calle de l'Irlandais à la foire: frapper partout où l'on voit une tête. J'y mets les formes, bien entenda, et toute ma faible prudence; mais je veux dire qu'il faut que l'on sache qui a tort et qui a raison.

Il m'a fallu beaucoup écrire, sur des affaires souvent où, laissé à moimême, je n'aurais rien su dire de bon; mais j'ai comme vous le savez, les volumes du $N$. Monde sous la main et e'est pour moi un véritable arsenal. Cela me permet de parler de toutes les choses en litige avec une grande précision et surtout de donner des preuves parfois absolument irrécusables. Nos adversaires sont très vagues et ces preuves détaillées les ruinent. Voici copie du Sommaire de l'un des Mémoires que j'ai faits pour répondre aux bons libéraux q'ii travaillent ici de leur côté. L'ouvrage a 182 pages, et peut se résumer comme suit:

Dans la 1ère Partie, on prouve que la position faite à l'Eglise et aux catholiques dans la Confédération Canadienne n'est nullement cet état précaire et si peu honorable que les Catholiques-libéraux se plaisent à dire, pour arracher au Clergé des concessions déraisonnables, ou le faire taire, en le menaçant de la haine des protestants. La Constitution même soustrait l'Église, sa liberté et ses droits acquis à tout danger de persécution, ou d'intervention dans nos affaires religieuses $d \epsilon$ la part de ces derniers.

Dans la 2nde partie, on prouve que ce sont les catholiques libéraux qui cherchent à réveiller le fanatisme des protestants contre le Clergé et ses amis qui dominent dans la Province de Québec, et que les protestants, même ainsi provoqués, sont loin d'accepter le rôle odieux que les libéraux veulent leur faire jouer, en les introduisant dans nos luttes intestines.

Dans la Sème partie, on prouve qu'il existe en Canada tout un parti de véritables libéraux, ou d'hommes imbus de ces erreurs astucieuses, d'autant plus dangereuses, comme z'exprime le Saint-Père dans son Bref, 
qu'elles trompent beaucoup de personnes honnêtes et les entraînent à s'écarter de la saine doctrine, surtout dans les questions qui, à première vue, semblent être du ressort du pouvoir civil plutôt que du pouvoir clésiastique; elles affaiblissent la Foi, elles rompent les liens de l'unité, elles divisent les forces catholiques et donnent une aide très efficace aux ennemis de l'Église.

Lo parti libéral n'est pas seulement un parti politique. Il professe dans ses journaux et par les discours de ses chefs, des maximes fausses en Religion et une politique opposée aux droits et aux intérêts de l'Église. Si ce parti compte encore des catholiques sincères, ces derniers ne le conduisent pas, mais sont conduits et exploités par des chefs hostiles au Clergé, aux Evêques et au Saint-Siège lui-même.

(Tout ce qui a été dit par la presse du parti libéral, l'Avenir, le Pays, le National, l'Evénement, le Journal de Québec et le Bien Public, de plus significatif et de plus compromettant pour eux, est cité ici).

Dans la 4ème partie enfin, on rappelle ce que l'Épiscopat, le Clergé et les vrais enfants de l'Église ont dû faire pour contrôler les libéraux de toutes nuances. Dans leurs Mandements, leurs Lettres Pastorales et les Décrets de leurs Conciles Provinciaux, les Évêques du Canada opposèrent une forte barrière aux envahissements des erreurs du libéralisme. Conformément aux désirs du Saint-Père, ceux d'entre eux qui le purent fondèrent une forte presse catholique, qu'ils opposèrent aux agressions de la presse libérale et aux entreprises dangereuses de quelques hommes politiques sur les droits sacrés de l'Église. A cette action du Clergé se joignirent les efforts d'une phalange de laïques absolument dévoués aux principes du Saint Siège.

La politique ne tarda pas à devenir, dans la Province, plus franchement conservatrice et chrétienne que jamais. Il sortit de leur programme un gouvernement fort, qui opéra dans nos lois les réformes les plus salutaires et j'attira par ses principes la confiance et l'appui du Clergé et de la très grande majorité des Catholiques de la Province. Ce fut au milieu des luttes pour en arriver là, que les amis des vrais principes ultramontains et Romains eurent le chagrin de voir, en plus d'une occasion difficile, Mgr. l'Archevêque de Québec leur faire absolument défaut et pousser même la sévérité contre eux jusqu'à chercher à les écráser sous le poids de son autorité. Ses lettres publiques l'attestent.

Ce cadre, assez étendu, m'a permis d'y faire entrer à peu près tout ce que j'avais à dire, au sujet de nos libéraux de principes, des manœuvres de leurs amis tant dans l'Église que dans l'État, et des bons combats soutenus pour la défense de la vraie doctrine et de la bonne cause Ultramontaine.

J'ai la satisfaction de vous dire que le tout a été lu, avec une attention spéciale, par quelques-uns du moins des Cardinaux de la Propagande, et que la chose a produrt le meilleur effet lans leur $\epsilon$ sprit. Je continuerai ce travail d'informations jusqu'au bout, ou tant que les Éminents Cardinaux qui me font l'honneur de me recevoir et de m'encourager le désireront. Ces choses doivent rester dans notre cercle; mais il est bon qua vous sachiez 
parfaitement ce que je fais et soutiens ici. J'ai beaucoup aimé les écrits du $N$. Monde de ces derniers təmps et j'ai passé au Cardinal Oreglia les articles sur l'influence indue cléricale, qui m'ont paru si dignes d'éloges. Soyons toujours prudents, mais fermes et courageux.

Veuillez, mon cher ami, agréer pour vous-même et offrir à nos excellents collaborateurs et amis l'expression des sentiments distingués avec lesquals j'ai l'honneur d'être

Votre très humble et to:ıt dévoué serviteur

M.A. Desjardins, M.P.

G. Lamarche, P.D.

Montréal P.Q., Canada

(M. l'abbé Joseph-Uldéric Leclere, curé de St-Vincent-de-Paul, (Ile Jésus) à M. Alphonse Desjardins).

mars 1874

..."Vos remarques sur le N. Monde n'ont fait que me confirmer dans les idées que j'avais moi-même. J'en avais parlé à l'Êvêque, jeudi dernier, et j'ai hâte de vous voir pour savoir ce qu'il y aurait à faire. Dans mon opinion, les hommes à la tête de la rédaction ne sont pas ce qu'il faut, y compris le censeur. L'Évêque serait prêt à nous seconder, car il sent luimême qu'il y a quelque chose qui boîte quelque part... Comme vous êtes au centre des affaires, (à Ottawa), et que vous êtes mieux à portée de juger que moi, faites en sorte de donner au journal une tournure aussi dégagee que possible de tout esprit de partie (sic)... Avec le temps, je crois qu'il faudra de toute nécessité que M. Lamarche cesse d'habiter le Bureau du $N$. Monde. Je crois que sa présence continuelle et son immixtion dans toutes les affaires du Journal sont une cause d'embarras et d'éloignement de plusieurs. Mais pour cela, il faudrait un Rédacteur plus indépendant des parties (sic) que M. Beausoleil, et qui pût mieux représenter les idées du Journal..."

(Extraits de La Lanterne, publiée en 1884).

P. 89: "...Je sais bien, écrit Buies, que le Nouveau-Monde voudrait avoir un dieu à lui tout seul. Majs ce n'est pas la peine d'avoir créé les mondes, de régner dans l'espace, d'être infini par la durée, pour venir s'enfermer dans un atelier de bedeaux. - Quand le Nouveau-Monde aura prouvé que son Dieu est le seul vrai, alors il sera temps de mettre devant sa porte une statue avec cette inscription: Hic est Lamarchi Deus qui fecit coelum et terram, et omnia quae vadant, propterea (pour propter, 6videmment), Novum Mundum, suae delectationis operam"...

P. 127: “...J'apprends à l'instant que messire Lamarche va établir son confessionnal au bureau du Nouveau-Monde. - Les âmes timorés, qui s'y rendront faire leur meâ culpâ, n'auront l'absolution qu'à la condition de s'abonner à son journal. - A celles qui sont encore un peu rétjves il infligera en outre, pour lour pénitenç, trois colonnes du Nouveau-Monde à lire tous les jours..." 